\title{
Calculating Machine Solution of Quadratic and Cubic Equations by the Odd Number Method
}

1. Introduction. EAstLACK ${ }^{1}$ has published a method for the solution of quadratic equations by means of a calculc.ting machine. His process is extended here to the solution of cubic equations. In the ordinary manual operation of calculating machines, the use of the method of solving cubic equations presented here will not be found to be as convenient as the use of certain other methods, such as that of Newton. The method is described here, however, in the belief that it may find application in large scale, automatic computing machines (such as the IBM Sequence Controlled Calculator or the ENIAC) where a large number of operations is not objectionable, provided that the operations are repetitive and sufficiently simple. We limit our discussion to real roots. Eastlack's method of solving quadratic equations is first reviewed so that the extension of the method to cubic equations may be clearer.

2. Extraction of square roots by the odd number method. The ordinary method for finding on the calculating machine the square root of a number of 3 or 4 digits of the form

$$
(10 x+y)^{2}=100 x^{2}+20 x y+y^{2}
$$

consists in removing the $100 x^{2}$ term by subtracting the $x$ odd numbers, $1,3,5, \cdots,(2 x-1)$, from the first one or two digits of $(10 x+y)^{2}$, and then removing the remainder of $(10 x+y)^{2}$ by subtracting the $y$ numbers, $(20 x+1),(20 x+3), \cdots,(20 x+2 y-1)$. This process of square root extraction may evidently be extended to the evaluation of roots having more than two digits.

The odd number method of square root extraction and a process of division may be combined expeditiously on a calculating machine to solve quadratic equations, of which we consider two types.

3. Positive root of $a=b x+x^{2}$ where $a, b>0$. This quadratic equation has one positive root and one negative root. To illustrate the calculating machine solution of this equation we find the first six digits of the positive root of $3586=80 x+x^{2}$. The successive steps of the solution are given in Table I. The number $a=3586$ is entered on the machine as if for square root. Then from it are subtracted as in that process the numbers $1,3,5, \cdots$, each however combined with $b=80$. The selection of columns is as follows: If the number $b$ is placed under $a$ so that their corresponding powers of 10 are aligned, the numbers $1,3,5, \cdots$, appear under the units column of $a$. If $b$ is moved $k$ places from this position, then the odd integers are moved $2 k$ places in the same direction, so that they always appear under the righthand digit of one of the pairs of digits in $a$. Of course $k$ is taken as large algebraically as possible without the combined subtrahend exceeding $a$. Just as in square root, the subtrahend is increased by 2 before each subtraction, but when the next subtraction would produce a negative result the 2 is replaced by 1,1 is added in the next column to the right, to whatever digit of $b$ is already there, and the carriage is shifted. Just as in ordinary square root the successive digits of the result appear in the counting dials. As in 
square root also when more than half the desired number of digits of the result have been obtained one may obviously cease to append any new sets of odd digits to the subtrahend and let the process degenerate into division.

TABLE I. Positive root of $3586=80 x+x^{2}$

$\begin{array}{ccccc}\begin{array}{c}\text { Keyboard } \\ (1)\end{array} & \begin{array}{c}\text { Counting Dials } \\ (2)\end{array} & \begin{array}{c}\text { Adding Dials } \\ (3)\end{array} & \begin{array}{c}\text { Digit Added to } \\ \text { Keyboard }\end{array} & \begin{array}{c}\text { Accumulation of } \\ \text { Column (4) } \\ (5)\end{array} \\ 080 & & 3586 & 01 & 01 \\ 090 & 1 & 2686 & 02 & 03 \\ 110 & 2 & 1586 & 02 & 05 \\ 130 & 3 & 286 & 01 & 06 \\ 140 & 3 & 286 & 001 & 061 \\ 141 & 31 & 2 & 002 & 063 \\ 143 & 32 & 20000 & 001 & 064 \\ 144 & 320 & 5599 & 00001 & 06401 \\ 14401 & 3201 & 145 & 00001 & 06402 \\ 14402 & 32013 & 12624 & & \\ 14402 & 320138 & & & \end{array}$

4. Smaller positive root of $a=b x-x^{2}$ where $a, b \geqq 0$ and $4 a<b^{2}$. This quadratic equation has two positive roots. Quadratic equations having two negative roots may be changed to this form by changing the signs of the roots. The smaller root may be obtained by the process outlined in 3 , with the exception that the odd numbers, $1,3,5$, etc., are this time to be subtracted from the number $b$ which is set on the keyboard. The subtraction of these odd numbers at no stage causes the number on the keyboard to become negative since $b>2 x$ if $x$ is the smaller of the two positive roots.

5. Extraction of cube roots by the odd number method. Suppose that $10 x+y$ is an integer of two digits, $x$ being the first digit and $y$ the second digit. Then

$$
(10 x+y)^{3}=1000 x^{3}+300 x^{2} y+30 x y^{2}+y^{3}
$$

may be regarded generally as an integer of six digits if in particular cases the first one or two of the six digits are zero. Now the portion of the first triad, or group of three digits arising from the term $1000 x^{3}$ may be represented by the series

$$
1000 x^{3}=1000\left[1+(1+6)+(1+6+2 \cdot 6)+\cdots+\left(3 x^{2}-3 x+1\right)\right] \text {, }
$$

having $x$ terms. We may thus determine $x$ by subtracting successively from the first group of 3 digits, $1,7,19,37, \cdots, 3 x^{2}-(3 x-1)$, the number $x$ of subtractions being recorded in the counting dials. The subtrahends themselves are built up by adding successively to the original 1 the numbers $6,12,18, \cdots, 6(x-1)$, the number added being always 6 times the number currently in the counting dials. When $1000 x^{3}$ is thus removed the remainder is $300 x^{2} y+30 x y^{2}+y^{3}$. For values of $y=1,2, \cdots$, this has the successive values $300 x^{2}+30 x+1,600 x^{2}+120 x+8,900 x^{2}+270 x+27$. We may therefore find $y$ by subtracting successively the initial value $300 x^{2}+30 x+1$, and the consecutive differences, $300 x^{2}+90 x+7,300 x^{2}+150 x+19, \cdots$, these successive subtrahends being built up by adding to the original $300 x^{2}+30 x+1$ on the keys the successive values $60 x+6,60 x+12, \cdots$, $60 x+6(y-1)$, this number being 6 times that currently present on the counting dials. The initial value in this sequence of subtrahends is obtained from the final value of the earlier sequence. The latter is $1000\left(3 x^{2}-3 x+1\right)$. To this we add $1000(3 x-1)$ and get $3000 x^{2}$. Shif ting the carriage one place to the left makes this effectively $300 x^{2}$, and to this we add $30 x+1$ to get 
the first subtrahend of the new sequence. This process of cube root extraction may evidently be extended to evaluate roots having more than two digits.

We apply this method of cube root extraction to the solution of three types of cubic equations in which the quadratic term has been removed.

6. Smaller positive root of $a=b x-x^{3}$ where $a, b>0$ and $27 a^{2}<4 b^{3}$. This cubic equation has three real roots, two of which are positive and one negative. Similar cubic equations having two negative roots and one positive root may be transformed to this form by changing the signs of the roots. To illustrate the calculating machine solution of this equation we find the first six digits of the smaller positive root of $95242=4000 x-x^{3}$. The successive steps of the solution are given in Table II. As in the quadratic case the constant term $b$ is combined on the keys with the variable subtrahend which is to erase $x^{3}$. Since the latter is built up from its differences the term $b$ is merely put into the first value and remains there. In this particular type of equation the cubic term has a minus sign. Hence the successive differences are subtracted from, instead of being added to the subtrahend. The rule for placing the columns is very similar to the quadratic case. If $b$ has its columns aligned with the corresponding ones in $a$, then the numbers $1,7,19, \cdots$ are in the units place. If $b$ is moved $k$ columns from that position then these numbers are moved $3 k$ columns in the same direction. As in the quadratic case $k$ is initially given the largest value that leaves the subtrahend less than $a$. When a digit in the result is obtained, the carriage is moved one place to the left. This moves $b$ in effect one place to the right compared to the residue of $a$. The new sequence $1,7,19, \cdots$ is then put in on the keys two places to the right, and hence three places to the right compared to $a$. The process of subtracting multiples of the partial root from the keyboard is most easily performed digit by digit, so that it is never necessary to subtract a number greater than 54. At the stage when 3201 appears on the counting dials, four of the required six digits of the root have been obtained, and two more digits may be obtained correctly by dividing the remainder 0.729601 on the carriage by the number 926.0797 appearing on the keyboard. Thus $x=32.0107$ is the smaller positive root to six digits. The remaining roots are most conveniently obtained by eliminating this root and solving the resulting quadratic equation.

TABLE II. Smaller positive root of $95242=4000 x-x^{3}$

\begin{tabular}{lcclc}
$\begin{array}{c}\text { Keyboard } \\
(1)\end{array}$ & $\begin{array}{c}\text { Counting Dials } \\
(2)\end{array}$ & $\begin{array}{c}\text { Adding Dials } \\
(3)\end{array}$ & \multicolumn{2}{c}{ Digits Subtracted from Keyboard } \\
4000 & & 95242 & 01 & $(4)$ \\
3900 & 1 & 56242 & 06 & $=6$ Col. (2) \\
3300 & 2 & 23242 & 12 & $=6$ Col. (2) \\
2100 & 3 & 2242 & 08 & $=3$ Col. (2) -1 \\
1300 & 3 & 2242 & 0091 & $=30$ Col. (2) +1 \\
1209 & 31 & 1033 & 0186 & $=6$ Col. (2) \\
1023 & 32 & 10 & 0095 & $=3$ Col. (2) -1 \\
0928 & 320 & 10000000 & $00009601=30$ Col. (2) +1 \\
09270399 & 3201 & 729601 & $00009602=3$ Col. (2) -1 \\
09260797 & 320107 & 81345 & &
\end{tabular}

Note that the subtraction of the terms of the odd number sequence at no stage causes the number on the keyboard to become negative since $b>3 x^{2}$ if $x$ is the smaller of the two positive roots.

7. Real root of $a=b x+x^{8}$ where $a, b>0$. This equation has one real root which is positive. Similar cubic equations having but one real root 
which is negative may be transformed to this form by changing the signs of the roots. The root may be obtained by the process outlined in 6 , with the exception that the terms of the odd number sequence, $1,(1+6)$, $(1+6+2 \cdot 6)$, etc., are this time to be added to the number $b$ which is set on the keyboard.

8. Real root of $a=x^{3}-b x$ where $a, b \geqq 0$ and $27 a^{2}>4 b^{3}$. This cubic equation has one real root which is positive. Similar cubic equations having but one real root which is negative may be transformed to this form by changing the signs of the roots. To illustrate the calculating machine solution of this type of cubic we find the first six digits of the real root of $32541=x^{3}-9 x$. The successive steps of the solution are given in Table III. The number $a=32541$ is registered on the adding dials. Set the number 100 on the keyboard, placing the unit hundreds digit, which is the first member of the odd number sequence, $1,(1+6),(1+6+2 \cdot 6)$, etc., in the column beneath the digit 2 of 32541 on the carriage. The number $b=9$ is subtracted from the keyboard, giving a new number 91 which is subtracted from the carriage. The process of adding terms of the odd number sequence to $-b$ on the keyboard, and subtracting the resulting numbers from the remainder on the carriage is now continued as indicated in Table III. The real root is $x=32.0199$, the last two digits being obtained by the division process described in 6 .

TABLE III. Real root of $32541=x^{3}-9 x$

\begin{tabular}{|c|c|c|c|c|}
\hline Keyboard & Counting Dials & Adding Dials & Digits A & Added to Keyboard \\
\hline (1) & (2) & (3) & & (4) \\
\hline$(-0009)$ & & 32541 & 0100 & $=6 \mathrm{Col}(2)$ \\
\hline $\begin{array}{l}0091 \\
0691\end{array}$ & $\begin{array}{l}1 \\
2\end{array}$ & $\begin{array}{l}31631 \\
24721\end{array}$ & $\begin{array}{l}0600 \\
1200\end{array}$ & $\begin{array}{l}=6 \text { Col. (2) } \\
=6 \text { Col. (2) }\end{array}$ \\
\hline 1891 & 3 & 5811 & 0800 & $\begin{array}{l}=3 \text { Col. (2) }-1 \\
=30 \text { Col (2) }+1\end{array}$ \\
\hline $\begin{array}{l}2691 \\
2782\end{array}$ & 31 & $\begin{array}{l}5811 \\
3029\end{array}$ & $\begin{array}{l}0091 \\
0186\end{array}$ & $=6$ Col. (2) \\
\hline & & & 0095 & $=3$ Col. (2) - \\
\hline 3063 & 320 & & 0000960 & $\begin{array}{l}=30 \text { Col. (2) } \\
=3 \text { Col. (2) }\end{array}$ \\
\hline 203 & $\begin{array}{l}3201 \\
32019\end{array}$ & $\begin{array}{r}30360399 \\
2776116\end{array}$ & 602 & \\
\hline 03 & 320199 & 17688 & & \\
\hline
\end{tabular}

In the above illustration the number $b$ was taken small enough relative to the number $a$ so that the first term of the odd number sequence was greater than $b$, and hence could be combined with $-b$ on the keyboard. It is evident, however, that $b$ may be greater than several terms of the odd number sequence even though $3 x^{2}>b$ where $x$ is the real root. In this event it is necessary to subtract several terms of the odd number sequence from the carriage and independently add the number $b$ to the carriage the same number of times, until a term of the odd number sequence is arrived at which is greater than $b$, and hence can be combined with $-b$ on the keyboard. The digits of the root will appear correctly on the counting dials, if zero is removed from the carriage every time that $b$ is added to the carriage.

W. E. BLEICK

Naval Postgraduate School,

U. S. Naval Academy,

Annapolis, Md.

1 ALLEN C. EASTLACK, [Methods of approximating roots of a quadratic equation, with a calculating machine], Amer. Inst. Actuaries, Record, v. 24, 1935, p. 21-23. 